\title{
Questes
}

\section{La prophétie d'Anselme de Laon à l'Anonyme poitevin (XIe-XIIe siècle)}

\section{Maria Valeria Ingegno}

\section{(2) OpenEdition}

\section{Journals}

\section{Édition électronique}

URL : http://journals.openedition.org/questes/3435

DOI : 10.4000/questes.3435

ISSN : 2109-9472

\section{Éditeur}

Les Amis de Questes

\section{Édition imprimée}

Date de publication : 15 juin 2014

Pagination : 29-52

ISSN : 2102-7188

\section{Référence électronique}

Maria Valeria Ingegno, "La prophétie d'Anselme de Laon à l'Anonyme poitevin (Xle-Xlle siècle)», Questes [En ligne], 28 | 2014, mis en ligne le 15 juin 2014, consulté le 02 mai 2019. URL : http:// journals.openedition.org/questes/3435; DOI : 10.4000/questes.3435 


\title{
La prophétie d'Anselme de Laon à l'Anonyme poitevin $\left(\mathrm{XI}^{\mathrm{e}}-\mathrm{XII}^{\mathrm{e}}\right.$ siècle $)$
}

\author{
Maria Valeria INGEGNO \\ Pontificia Università Gregoriana - E.P.H.E.
}

\section{Les études menées sur la théorie de la prophétie en relation avec le Moyen Âge scolastique : un excursus bibliographique}

La théorie de la prophétie, telle qu'elle est présentée dans les traités produits dans le domaine scolastique, bénéficie d'études brillantes : au cours des dernières décennies les historiens et les philosophes de la connaissance ont redonné de l'importance à ce thème. En 1938, dans un long article publié dans le Dictionnaire de Théologie Catholique, Alain Michel se montrait encore principalement engagé dans la définition de la prophétie et dans la légitimisation de son emploi. Son étude rappelait simplement ipso facto quelques personnalités caractéristiques du Moyen Âge central impliquées dans l'emploi de l'argument de la prophétie contre les Juifs ${ }^{1}$. C'est donc à Bruno Decker que revient le mérite d'avoir, en

${ }^{1}$ Cf. Alain Michel, art. «Prophétie », Dictionnaire de Théologie Catholique, t. XIII, Paris, 1936, col. 708-737, en particulier col. 712. Gilbert Dahan reviendra sur le thème des rapports entre Juifs et Chrétiens, en soulignant pour le XII ${ }^{\mathrm{e}}$ et surtout le $\mathrm{XIII}^{\mathrm{e}}$ siècle l'emploi, dans un contexte chrétien, d'une exégèse hébraïque employée en vue d'offrir une lecture des textes prophétiques. L'auteur soulignera en effet plus précisément la contribution offerte par la réflexion de Maïmonide à l'exégèse d'Albert le Grand, mais aussi la polémique menée contre les Juifs par des auteurs tels que Guerric de Saint-Quentin, Guillaume d'Alton, Albert le Grand lui-même, Hugues de Saint-Cher, Pierre de Jean Olieu (via Jérôme), et l'impulsion favorable à l'exégèse médiévale née de cet affrontement ( $c f$. Gilbert Dahan, "L'utilisation de l'exégèse juive dans la lecture des livres prophétiques au XIII ${ }^{\mathrm{e}}$ siècle », dans Neue Richtungen in der hoch-und spätmittelalterlichen Bibelexegese, dir. Robert E. Lerner, München, Schriften des Historischen Kollegs. Kolloquien, 1996, p. 121-138. 
1940, su ouvrir la voie aux études sur le sujet à partir d'une réflexion portant aussi bien sur le thème de la révélation que sur celui de la prophétie $^{2}$. Grâce à sa bronographie, ce spécialiste du Moyen Âge n'a pas mis en relief le contenu d'une révélation en particulier, mais bien la théorie de la révélation prophétique telle qu'elle était présente chez les magistri du $\mathrm{XIII}^{\mathrm{e}}$ siècle $^{3}$. Les textes d'Augustin, de Grégoire le Grand et d'Isidore de Séville sont ainsi présentés comme des textes propédeutiques préparant la réflexion des grands auteurs de la Scolastique ${ }^{4}$, que Bruno Decker, grâce à la présentation d'un ensemble inédit, identifie en les personnes de Guillaume d'Auxerre, Guillaume d'Auvergne, Philippe le Chancelier, Alexandre de Halès, Albert le Grand, Bonaventure et surtout Thomas d'Aquin ${ }^{5}$. Une partie de l'œuvre permet au lecteur de comparer cette production et la contribution de plus en plus importante de la culture hébraïque et surtout arabe ${ }^{6}$.

\section{Les études relatives à Thomas d'Aquin et Hugues de Saint- Cher}

Après les premiers pas de Bruno Decker, le thème des études portant sur la théorie de la prophétie au XIII ${ }^{\mathrm{e}}$ siècle s'est révélé particulièrement fructueux pour les chercheurs, notamment en ce qui concerne la personne et la production de Thomas d'Aquin, auteur, comme on le sait, d'une Summa theologica ${ }^{7}$ ainsi que d'un De prophetia au sein de son De veritate $(\text { Quaestio XII })^{8}$.

\footnotetext{
${ }^{2}$ Bruno Decker, Die Entwiclung der Lehre von der prophetischen Offenbarung von Wilhelm von Auxerre bis zu Thomas von Aquin, Breslau, Müller und Seiffert, 1940.

${ }^{3}$ Ibid., p. 1.

${ }^{4}$ Ibid., p. $5-13$.

${ }^{5}$ Ibid., p. 47-208.

${ }^{6}$ Ibid., p. 13-38.

${ }^{7}$ Thomas d'Aquin, Summa theologiae et summa contra gentiles, Romae, Commissionis Leoninae S. Sabina, 1948.

${ }^{8}$ Thomas d'Aquin, Questions disputées sur la vérité. Question XII. La prophétie (De prophetia), éd. Jean-Pierre Torrell, Paris, Vrin, 2006.
} 
Après une première contribution de Serafino Zarb destinée à identifier les sources augustiniennes de la Summa ${ }^{9}$, puis une importante intervention de Bruno Decker lui-même portant aussi bien sur la Summa que sur les traités d'auteurs précédant Thomas ${ }^{10}$, c'est René Latourelle qui a traité brièvement, mais de manière claire et synthétique, le thème de la révélation prophétique telle qu'elle se présente dans la réflexion du Docteur angélique. Le chercheur a ainsi souligné combien le rappel constant des Écritures et l'importance psychologique prise par le divin dans la dimension humaine sont des caractéristiques propres de Thomas ${ }^{11}$. En 1967, Jean Richard analyse toutefois la Summa plus en détail: les modalités de la connaissance indiquées comme propres au prophète (visio corporalis, spiritalis et intellectualis) feraient référence à la réflexion du De Genesi ad litteram libri XII d'Augustin ${ }^{12}$, sans pour autant que ne soient exclues une réflexion sur la species (Aristote) et une distinction entre l'acceptio et le judicium d'ordre surnaturel ${ }^{13}$. Ce n'est que trente ans plus

9 Serafino Zarb, «Le fonti agostiniane del trattato sulla profezia di S. Tommaso d'Aquino », Angelicum, 15, 1938, p. 169-200.

${ }^{10}$ Bruno Decker, « Die Analyse des Offenbarungsvorganges beim hl. Thomas im Lichte vorthomistischer Prophetientraktate », Angelicum, 16, 1939, p. 195-244.

11 René Latourelle, Théologie de la Révélation, Paris, Desclée de Brouwer, 1963, p. 171-177.

12 Pour une analyse de la théorie de la prophétie telle qu'elle est exprimée chez Augustin, non seulement dans le De Genesi ad litteram libri XII, mais également dans le De ciuitate Dei, voir Robert Austin Markus, Saeculum. History and Society in the Theology of St. Augustin, Cambridge, Cambridge University Press 1970, p. 187-196.

13 Jean Richard, «Le processus psychologique de la révélation prophétique selon saint Thomas d'Aquin », Laval Théologique et philosophique, 23, 1967, p. 42-75. Selon Serge Bonino, le De veritate indiquerait plutôt qu'une dimension surnaturelle élève la nature humaine de l'intérieur, sans la modifier: la révélation divine, entendue comme un don, s'inscrirait en effet dans la dynamique naturelle de la connaissance humaine et en respecterait les modalités ( $c f$. Serge-Thomas Bonino, "Le rôle de l'image dans la connaissance prophétique d'après saint Thomas d'Aquin », Revue Thomiste, 89, 1989, p. 533-568). Selon le chercheur, le processus de la révélation n'impliquerait donc pas la présence de species dans l'intellect ou l'imagination, mais une lumière prophétique qui renforce et perfectionne la lumière naturelle de l'intellect agissant, sans le remplacer. L'intellect du prophète, activé par le lumen propheticum, deviendrait ainsi capable d'extraire des fantômes provenant des sens 
tard, dans sa longue introduction à l'édition de la Summa, que Jean-Pierre Torrell mettra en évidence certains thèmes majeurs de la réflexion du magister, c'est-à-dire l'essence de la prophétie, sa cause, les modalités de la connaissance prophétique et enfin ses différentes formes ${ }^{14}$. L'érudit identifie dans la conception de l'acte prophétique par Thomas d'Aquin une illumination provisoire de l'esprit et une forme de connaissance dans le cadre d'« une réflexion sur les structures de l'esprit humain et sur sa place dans l'univers des intelligences, sur la philosophie de la connaissance et le rôle respectif de la raison et de l'imagination $[\ldots]^{15} »$. Dans le cadre du processus psychologique en question, le rôle de Dieu et de l'homme seraient analysés sans pour autant être séparés, tandis qu'une distinction serait établie entre ce qui dérive de la lumière concédée par inspiration divine (lumen) et les représentations (ou species) qui constituent la matière.

La Quaestio XII. De prophetia du prince de la scolastique a été associée à la Quaestio du même titre qui en constitue la source, et que l'on attribue à Hugues de Saint-Cher en raison du manuscrit de Douai $434^{16}$. En 1974, Jean-Pierre Torrell avait déjà souligné que les deux auteurs avaient en commun la définition de la prophétie comme une forme d'habitus (et non de passio $)^{17}$, ainsi qu'un intérêt plus général envers le thème de la connaissance prophétique en elle-même. Selon Hugues, cette connaissance peut être obtenue par le biais d'une réalité créée, tandis que Thomas, en

des species porteuses d'une vérité plus élevée, que l'on peut définir surnaturelle (ibid., p. 566-567). Pour une étude attentive du lexique propre à la prophétie tel qu'il apparaît non seulement dans la Summa theologiae $I I^{a}-I I^{a e}$ mais également dans la Quaestio XII. De veritate, voir en revanche Alessandro Ghisalberti, « Il lessico della profezia in s. Tommaso d'Aquino », Cristianesimo nella Storia, 17, 1996, p. 349368.

${ }^{14}$ Thomas d'Aquin, Somme théologique. La prophétie $2^{a}-2^{a e}$, Questions 171-178. trad. Pierre Synave-Paul Benoît, intr. Jean-Pierre Torrell, Paris, Éditions du Cerf, 2005, p. 33-98.

15 Ibid., p. 9.

16 Jean-Pierre Torrell, «Hugues de Saint-Cher et Thomas d'Aquin. Contribution à l'histoire du traité de la prophétie », Revue Thomiste, 74, 1974, p. 5-22.

${ }^{17}$ Ibid., p. 7. 
héritier d'Aristote, y voit une conséquence de l'action de Dieu dans l'âme du prophète ${ }^{18}$. Le chercheur est ensuite revenu sur la réflexion d'Hugues à l'occasion de son édition de la Quaestio 481 du manuscrit de Douai, qui lui est attribuée ${ }^{19}$. Ici, la prophétie serait conçue selon la définition établie par Cassiodore, comme une forme d'inspiratio ou de revelatio et comme un acte transitoire (non pas comme un habitus, donc) ${ }^{20}$. Dans son œuvre, Hugues s'arrêterait longuement sur le spiritus en tant que partie de l'âme où s'impriment les images accompagnant la connaissance prophétique ${ }^{21}$, mais la réflexion du maître reconnaîtrait tout de même un rôle important à la fonction réceptrice de l'intellect ${ }^{22}$.

\section{Les autres auteurs du XIII ${ }^{\mathrm{e}}$ siècle}

$\mathrm{Au}$ cours de ses recherches, Jean-Pierre Torrell a présenté aux lecteurs des traités sur la prophétie rédigés par les maîtres les plus divers : de Philippe le Chancelier à Albert le Grand, du franciscain Jean de Roquetaillade à l'Anonyme du ms. Assise 186, ce dernier écrit étant peutêtre une œuvre de Bonaventure ${ }^{23}$. Sous la forme de quaestiones disputatae, ces auteurs proposeraient surtout une réflexion sur la connaissance des futurs contingents et une définition du rapport entre Dieu et l'homme centrée sur le lumen de la connaissance prophétique, définition influencée par la contribution d'Augustin. Les apports de Cassiodore, Grégoire le Grand, la Glose ordinaire et ses extraits, ainsi que d'Aristote, d'Avicenne

\footnotetext{
${ }^{18}$ Ibid., p. 16 ; p. 20.

${ }^{19}$ Théorie de la prophétie et philosophie de la connaissance aux environs de 1230. La contribution d'Hugues de Saint-Cher (Ms. Douai 434, Question 481). Édition critique avec introduction et commentaire par Jean-Pierre Torrell, Louvain, Spicilegium Sacrum Lovaniense, 1977.

${ }^{20}$ Ibid., intr., p. 153-156.

${ }^{21}$ Ibid., intr., p. 170-187.

${ }^{22}$ Jean-Pierre Torrell, Recherches sur la théorie de la prophétie au Moyen Âge, op. cit., p. 220.

${ }^{23}$ Ibid., p. 47-74 ; p. 119-203 ; p. 231-50 ; p. 251-317.
} 
et d'Averroès - du moins en ce qui concerne Albert le Grand et Thomas d'Aquin -, ne sont pas pour autant exclus de cette importante réflexion ${ }^{24}$. Hélène Merle a toutefois proposé une distinction au sein de notre groupe de maîtres en fonction d'un thème particulier inhérent à la connaissance prophétique: il s'agit du speculum, présent chez certains sommistes du $\mathrm{XIII}^{\mathrm{e}}$ siècle. En adoptant cette image, certains auteurs, comme Guillaume d'Auxerre, l'Anonyme de Douai et Philippe le Chancelier, seraient en effet encore, de par leurs structures de pensée, des hommes du XII ${ }^{\mathrm{e}}$ siècle et des augustiniens faisant souvent référence à l'évêque d'Hippone ainsi qu'à l'œuvre pseudo-augustinienne De spiritu et anima ${ }^{25}$. En revanche, chez Thomas d'Aquin et Albert le Grand, qui connaitraient l'œuvre d'Aristote, d'Avicenne, d'Averroès et de Maïmonide, Hélène Merle relève une mentalité différente, plus intellectualiste et moins sensible à l'imaginaire biblique qui avait dominé jusque là : la pensée européenne passerait ainsi de l'imaginaire symbolique à l'abstraction noétique ou scientifique d'inspiration aristotélicienne, plus caractéristique du XIII ${ }^{\mathrm{e}}$ siècle $^{26}$.

Enfin, Marianne Schlosser a elle aussi abordé le thème de la prophétie $^{27}$. Dans sa monographie très approfondie, elle souligne, comme d'autres chercheurs, que les maitres universitaires et les philosophes du $\mathrm{XIII}^{\mathrm{e}}$ siècle tournent leur attention vers une définition du concept de la prophétie entendu comme une forme de connaissance ou en lien avec les futurs contingents. En ce qui concerne la connaissance prophétique et son annonce, l'auteur considère que la réflexion augustinienne, mais aussi (la réflexion) aristotélicienne, constituent le point de départ de ce raisonnement, tandis que le thème essentiel de la visio in speculo

\footnotetext{
${ }^{24}$ Ibid., p. 120-203 ; p. 205 ; p. 207; p. 220-24.

25 Hélène Merle, "Miroir de l'éternité et pierre spéculaire dans les traités sur la prophétie des XII ${ }^{\mathrm{e}}$ et XIII ${ }^{\mathrm{e}}$ siècles », AHDLMA, 59, 1992, p. 7-40, notamment p. 9-16.

${ }^{26}$ Ibid., p. 17-20.

${ }^{27}$ Marianne Schlosser, Lucerna in caliginoso loco. Aspekte des Prophetie-Begriffes in der scholastichen Theologie, Paderborn/München/Wien/Zürich, F. Schöningh, 2000.
} 
aeternitatis est traité en faisant plus particulièrement référence aux conditions relatives au raptus et à la contemplation ${ }^{28}$. Guillaume d'Auxerre, Hugues de Saint-Cher, Alexandre de Halès et l'Anonyme d'Assise sont cités en raison de leur questionnement sur la possibilité d'établir ou non une équivalence entre théologie et prophétie, tandis que Dominique et François sont définis comme des prophètes - selon la perception qu'en avaient leurs contemporains - en raison de leur activité de fondation d'un ordre ${ }^{29}$. Dans l'ensemble, ces auteurs se demandent surtout quel est le rôle de l'intellect dans le cadre du phénomène prophétique, si la prophétie constitue un don de la grâce sanctifiante ou un charisme et si, qu'elle soit le fruit d'un état conscient ou non, elle représente une condition momentanée ou une sorte de prédisposition.

\section{La production exégétique : un domaine inexploré}

Chez les auteurs scolastiques, au premier rang desquels Thomas d'Aquin, la théorie de la prophétie se révèle donc inhérente à un ensemble de réflexions d'ordre anthropologique et épistémologique particulièrement complexes, qui présentent des développements et des solutions distinctes, d'autant plus différentes que des doctrines aux origines et aux caractéristiques variées confluent vers l'Occident européen.

Dans l'introduction de ses Recherches sur la théorie de la prophétie au Moyen Âge, Jean-Pierre Torrell encourage donc les éditions et l'étude de ce type de traité ${ }^{30}$, sans souligner cependant comment l'intérêt suscité par les écrits de Thomas d'Aquin et d'autres auteurs de son siècle a, de fait, empêché les chercheurs d'explorer une grande partie de la production scolastique dans le domaine de l'exégèse plutôt que des traités et portant

\footnotetext{
${ }^{28}$ Ibid., p. $104-126$.

${ }^{29}$ Ibid., p. 206-226.

${ }^{30}$ Jean-Pierre Torrell, Recherches sur la théorie de la prophétie au Moyen Âge, op. cit., p. XIV-XV.
} 
(au moins en partie) sur le thème de la prophétie. Je fais référence aux commentaires de la $\mathrm{I}^{\text {ère }}$ Épître aux Corinthiens rédigés au cours du $\mathrm{XII}^{\mathrm{e}}$ siècle $^{31}$, les commentaires des Épîtres de Paul constituant un genre caractéristique de cette époque. En outre, en privilégiant, comme du reste tous les chercheurs, les auteurs à l'œuvre durant la période la plus active de la Scolastique - une seule étude de Torrell est consacrée à un auteur du $\mathrm{XII}^{\mathrm{e}}$ siècle, Pierre le Vénérable ${ }^{32}-$, le chercheur n'envisage pas la possibilité d'un approfondissement relatif à la réflexion des magistri de cette dernière période ${ }^{33}$.

Afin de combler ces lacunes, nous aborderons précisément le commentaire du chap. XIV, 1-40 de la I ${ }^{\text {ère }}$ Épître aux Corinthiens, tel qu'il a été proposé par quatre exégètes actifs entre le $\mathrm{XI}^{\mathrm{e}}$ et le $\mathrm{XII}^{\mathrm{e}}$ siècle. Dans ce contexte, la réflexion relative au charisme de la prophétie est souvent unie à celle sur le don de la polylalie (c'est-à-dire la capacité à parler plusieurs langues) et présente plusieurs types de développements.

\section{Anselme et Raoul de Laon}

Le premier texte que nous proposons d'examiner est le commentaire proposé par la Glose dite « ordinaire », que la tradition attribue aux maîtres Anselme et Raoul de Laon. Cette œuvre, dont l'importance se révèlera capitale dans le domaine de l'exégèse du XI ${ }^{\mathrm{e}}$ au XIII ${ }^{\mathrm{e}}$ siècle est composée de fait d'un ensemble de gloses de type marginal et interlinéaire portant sur le

31 Pour une vue d'ensemble de ce type de production, $c f$. Thomas d'Aquin, Commentaire de la première Épître aux Corinthiens, trad. Jean-Éric Stroobant de Saint Éloy, intr. Gilbert Dahan, Paris, Éditions du Cerf, 2002, p. XXXVIII-XL de l'introduction.

32 Jean Pierre Torrell, «La notion de prophétie et la méthode apologétique dans le Contra Saracenos de Pierre le Vénérable», dans Recherches sur la théorie de la prophétie au Moyen Age. XII $-X I V^{e}$ siècle, Fribourg, 1992, p. 75-100.

${ }^{33}$ Dans sa brève étude portant sur le statut de la prophétie, Gian Luca Potestà appelle lui aussi de ses vœux un élargissement du cadre d'étude portant également sur la production à caractère théologique : Gian Luca Potestà, «Lo statuto della profezia nel Medio Evo », Cristianesimo nella storia, 17, 1996, p. 243-250. 
texte biblique, qui occupe en revanche une place centrale dans le cadre d'une mise en page complexe. Il se révèle constitué d'une série d'extraits patristiques dont certains seulement sont mentionnés comme tels dans l'édition de Strasbourg d'Adolf Rusch ${ }^{34}$. Mises à part les sources signalées, nous avons pu identifier certaines citations remaniées qui semblent tirées du commentaire de l'Ad Corinthios prima de l'Ambrosiaster ${ }^{35}$ et de certaines œuvres d'Augustin ${ }^{36}$. Dans le domaine de l'exégèse, l'originalité des deux frères consiste à compléter les citations de la glose marginale par leurs propres commentaires et à rédiger la glose interlinéaire dans son intégralité.

Après une brève introduction, le De Genesi ad litteram ouvre la série des extraits marginaux : les deux frères y proposent une longue distinction entre les langues et la prophétie, distinction qu'ils ramènent à un schéma cognitif. Il s'agit pour eux, comme pour Augustin, de la distinction entre la mens et le spiritus de l'homme, cette première étant définie comme une force supérieure de l'âme qui nous donne la connaissance des choses ellesmêmes et ce dernier comme une force inférieure de la même âme dont la tâche consiste plutôt à recevoir les impressions sensibles des $\operatorname{choses}^{37}$. Augustin prenait pour exemple l'épisode de l'interprétation par Joseph du rêve du Pharaon concernant les sept épis et les sept boeufs : le Pharaon ne comprend pas et voit seulement in spiritu, Joseph en revanche voit in mente - c'est-à-dire qu'il comprend réellement et, de ce fait, prophétise. Dans le premier cas, on a affaire à la langue et à l'imagination des choses, dans le

34 Biblia latina cum Glossa ordinaria, éd. Karlfried Froelich, Margareth Gibson [réimpression en fac-simile de l'éd. de Strasbourg 1480-1481], Brepols, Turnhout, 1992.

35 Ambrosiaste, Ad prima Corinthios, éd. Heinrich Joseph Vogels, CSEL LXXXI, 2, Vindobonae, 1968.

36 Augustinus, De Genesi ad litteram libri XII, CSEL XXVIII, éd. Joseph Zicha, Pragae/Vindobonae/Lipsiae, 1894 et Épistula CLXIX, CSEL XLIV, éd. Alois Goldbacher, Vindobonae/Lipsiae, 1904.

${ }^{37}$ Glossa ordinaria, $\mathrm{f}^{0}$ 330. Cf. Augustinus, De Genesi ad litteram, XII, 8, p. 390, 1. 1923. 
deuxième cas à la prophétie et à l'interprétation de l'imagination. Dans tous les cas, c'est celui qui prophétise qui s'avère utile ${ }^{38}$. Dans une deuxième glose l'attention des deux frères porte sur les contenus de l'exposition paulinienne: ils examinent la prophétie par rapport à l'exposition des Écritures qui traitent des choses futures ou bien la voient comme la manifestation d'Isaïe et des autres prophètes, que l'esprit dévoile sans aucun concours humain ${ }^{39}$.

Un autre aspect est ensuite traité par le biais de l'exégèse de l'Ambrosiaster, là où les uelamina Legis ueteris qui conviennent aux indignes et aux infidèles sont opposés à la vérité transmise par le prophète et qui s'adresse aux fidèles ${ }^{40}$. Dans une deuxième glose, on traite plus explicitement du rapport entre les coutumes de la Loi, destinées aux infidèles et qui sont vehiculées par les langues, et le Nouveau Testament qui, sous forme de prophétie, s'adresse aux croyants, ou encore entre la révélation et les paraboles qui, elles, ont pour destinataires les indignes ${ }^{41}$.

${ }^{38}$ Glossa ordinaria, $\mathrm{f}^{\mathrm{o}}$ 330. Cf. Augustinus, De Genesi ad litteram, XII, 9, p. 391, 1. 4 24.

39 «Ut exponam scripturis quae de futuris agunt [...] prophetia est ut ysaiae vel veterorum quam sine humana industria spiritus sanctus paulatim reuelat »[...]. «Afin que je montre par des Ecritures qui traitent des choses futures [...] La prophetie est comme celle d'Isaïe ou des Anciens c'est-à-dire qu'elle est révélée progressivement par l'Esprit Saint, mais qu'elle ne l'est pas par un esprit humain » (Glossa ordinaria, $\mathrm{f}^{\mathrm{o}} 330$, nous traduisons).

40 Dicit linguas quibus velatur veritas mutandas quia etiam omnia velamina legis veteris dominus per prophetam esse mutanda dixit ut fidelibus perspicua fieret veritas remoto legalium figurarum velamine. Et hac similitudine ostendit, linguas quibus tegitur veritas non esse appetendas, nec dari propter fideles sed infideles, sicut in veteri lege velamen erat vt indignis et infidelibus velaretur veritas. "Il dit qu'il faut changer les langues qui cachent la vérité parce qu'ainsi a parlé le Seigneur par l'intermédiaire du prophète de telle sorte que la vérité se fasse voir une fois retiré le voile des préfigurations de la Loi. Et il montre par cette image qu'il ne faut pas chercher à atteindre les langues par lesquelles la vérité est cachée et qu'elle ne doit pas être donnée aux fidèles, mais aux mécreants, comme dans l'Ancienne Loi ou un voile était placé pour cacher la vérité aux indignes et aux mécreants " (Glossa ordinaria, $\mathrm{f}^{\mathrm{o}} 331$, nous traduisons. $C f$. Ambrosiaster, Ad Corinthios prima XIV, 22, p. 156, 1. 15-18).

${ }^{41}$ Non de sabbato et neomenijs et circumcisione et huiusmodi, sed nouum testamentum. Vel non in reuelatione qua digni sunt, sed in parabolis dicitur eis aeuangelium. 
Pour les deux frères - c'est évident - la prophétie consiste donc à exposer la vérité du Nouveau Testament: reste à savoir en quels termes. Comme Anselme et Raoul ne manquent pas de le souligner, la prophétie doit être utile sur le plan moral de l'aedificatio «quae necessaria est fidelibus ${ }^{42}$. La prophétie est utile aux fidèles «ut edificentur» et « habet aliquod donum quo potest uti ad edificationem ${ }^{43}$. Les deux maîtres spécifient à travers le texte de l'Epistula CLXIX d'Augustin, qui clôt les extraits de la glose marginale, que l'édification concerne la foi aussi bien que les mœurs: "de predictis quae ad fidem et mores aedificant ${ }^{44}$. Comme on le voit, leur réflexion à l'égard de certaines difficultés soulevées par le texte paulinien est donc motivée à plusieurs reprises par un intérêt fidéistique et moral.

À cet égard, Anselme et Raoul, dans une attitude conservatrice par rapport aux attitudes adoptées par les mouvements hérétiques de l'époque, soulignent, à la suite du texte paulinien et du verset de Genèse III, 16 que toute prise de parole de la part des femmes doit être bannie des églises : « [...] que les femmes gardent le silence, comme je l'enseigne, dans toutes les églises ${ }^{45} »$. En outre, les deux frères vont refusent également l'abandon de la tradition institutionnalisée et toute idée d'une quelconque innovation, en invoquant un verbum Dei dont l'Église est censée être la dépositaire et

« Non le Sabbat, les fêtes de la lune, la circoncision et les fêtes de ce genre, mais le Nouveau Testament. Ou non dans la révélation de laquelle ils sont dignes, mais dans ces paraboles est lue l'annonce" (Glossa ordinaria, $\mathrm{f}^{\circ} 331$, nous traduisons. $C f$. Ambrosiaster, Ad Corinthios prima XIV, 21, p. 155, 1. 19, p. 156, 1. 3).

42 «[...] les choses qui sont nécessaire aux fidèles; [...] pour édifier» (Glossa ordinaria, $\mathrm{f}^{\mathrm{0}} 331$, nous traduisons).

43 «Pour édifier il a qualque don qu'il peut employer pour l'édification» (Glossa ordinaria, $\mathrm{f}^{\mathrm{0}} 331$, nous traduisons).

44 «À l'égard des choses qui édifient la foi et les mœurs» (Glossa ordinaria, $\mathrm{f}^{\mathrm{0}} 332$, nous traduisons. $C f$. Augustinus, Epistula CLXIX, I, 2, éd. cit., p. 612, 1. 16-17).

45 «[...] mulieres taceant sicut ego doceo in omnibus aecclesijs » (Glossa ordinaria, $\mathrm{f}^{\mathrm{o}} 332$, nous traduisons). 
d'autres préceptes qui sont qualifiés de mandata $D e i^{46}$. Ils exaltent d'une manière significative les pusilli - les petits - qui sont plongés dans la contemplation du mystère de la Croix et qui, tout en ignorant ce dont on disserte subtilissime, n'en parviennent pas moins à la gloire grâce au sacrifice du Christ ${ }^{47}$. Après le rappel terrible du Jugement dernier pour ceux qui ne respectent pas les ordres divins ${ }^{48}$, la conclusion recommande à nouveau le silence aux femmes au nom de l'honnêteté et de l'ordre ${ }^{49}$.

46 «Increpatio, quasi suis institutionibus dimissis aliquas nouas vellent inducere, quod non possunt, quia ab eis non processit verbum dei, nec soli acceperunt. Et oportet vt ita cognoscat esse si quis est propheta vel spiritualis : alioquin si contra vadit, nec propheta nec spiritualis. Spirituales qui non prophetae sunt qui non habent noticiam de futuris ». "Voici un reproche, pour ainsi dire, abandonnées pour ainsi dire leurs institutions, ils voudraient en introduire de nouvelles, mais ils ne le peuvent, étant donné que la parole de Dieu ne vient pas d'eux, et que, seuls, ils ne lont pas accueillie. Et si quelqu'un est prophète ou spirituel, il est opportun qu'il sache quil en est ainsi : autrement, s'il va à l'encontre de ces institutions, il n'est ni prophète ni spirituel. Les spirituels sont ceux qui ne sont pas prophètes, qui ne connaissent pas les évènements futurs. » (Glossa ordinaria, $\mathrm{f}^{0} 332$, nous traduisons. $C f$. Augustinus, Epistula CLXIX, I, 2, éd. cit., p. 613, 1. 9).

${ }^{47}$ «Pusilli in cruce gloriantes et si ignorant quae subtilissime disseruntur, ad gloriam perueniunt, QUIA NON PERIT UNUS DE PUSILLIS PRO QUIBUS CHRISTUS MORTUUS EST». «Les petits qui se glorifient de la croix même s'ils ignorent ce qui est soutenu de manière extrêmement raffinée arrivent de même à la gloire, CAR UN PETIT POUR LEQUEL LE CHRIST EST MORT NE MEURT PAS » (Glossa ordinaria, $\mathrm{f}^{\mathrm{0}} 332$, nous traduisons).

48 «Sed si quis hec ignorat et non vult credere esse mandata dei ignorabitur vt in euangelio : NESCIO VOS, NON NOVI VOS ESSE MEOS, vel inter praedestinatos ». «Mais si quelqu'un ignore tout cela et ne veut pas croire que c'est l'ordre du Seigneur, il ne sera pas reconnu, comme dans l'Évangile : JE NE VOUS CONNAIS PAS, JE NE VOUS RECONNAIS PAS COMME MIENS, c'est-à-dire entre les prédestinés » (Glossa ordinaria, $\mathrm{f}^{0} 332$, nous traduison. Cf. Augustinus Epistula CLXIX, I, 2, p. 613, 1. 10-13).

49 «Non dicit de his qui non possunt discernere, ineffabilem trinitatis vnitatem, sed de praedictis quae ad fidem et mores aedificant, scilicet $v t$ linguis prophetia praeponatur, vt non perturbate geratur, quasi prophetiae spiritus etiam inuitos loqui cogat, vt mulieres taceant, vt omnia honeste et secundum hordinem fiant. Coercet ergo, et ad pacificum ordinem reuocat inquietos, tanto ad seditionem faciliores, quanto sibi videbantur spiritu excellentiores, cum superbiendo cuncta turbarent $»$. «Il ne fait pas référence à ceux qui ne peuvent comprendre l'ineffable unité de la Trinité, mais aux choses déjà citées qui édifient en vue de la foi et des coutumes, afin que la prophétie soit antéposée aux langues de sorte que ce phénomène ne soit pas géré de manière chaotique - pour ainsi dire, que l'esprit de prophétie oblige également à parler ceux qui ne le souhaitent pas - ainsi, que les femmes gardent le silence afin que tout se déroule avec dignité et ordre. Donc [1'Apôtre] contient et 
Ce texte latin relatif aux langues, mais surtout à la prophétie, a exercé une grande influence - ce n'est pas par hasard qu'Anselme de Laon a été appelé le "maître des maîtres ». Nous allons maintenant étudier les textes de trois auteurs poitevins qui reprennent le commentaire de la Glose en s'en éloignant en certains points d'importance.

\section{Jean de Poitiers}

Étudions à présent comment intervient notre deuxième auteur, l'inconnu Gratiadei, c'est-à-dire le magister Jean, actif à Poitiers aux alentours de 1102. Son identité et ses origines poitevines émergent indirectement du titre du manuscrit Paris, BnF. lat. 14442, $\mathrm{f}^{\mathrm{o}} 1 \mathrm{r}^{\mathrm{o}}:$ : In nomine patris et filii et spiritus sancti amen, incipiuntur glos[u]le epistolarum pauli ab illo videlicet cuius nomen gratiadei interpretatur in anno quo cons $[u] l$ pictauiensis de iherusolima redijt ». À la différence de la Glose, son commentaire, rédigé aux alentours de 1102, fait abstraction de toute évaluation gnoséologique et identifie la charité et la prophétie avec la prédication (en tant que premier fruit de l'amour) ${ }^{50}$. Jean en reconnaît ensuite les objectifs intrinsèques dans l'édification, l'exhortation et la consolation de 1 'homme ${ }^{51}$; en revanche, il en repère les contenus dans

rappelle à l'ordre et à la paix les inquiets, d'autant plus facilement sujets à la discorde qu'ils semblaient plus sublimes tandis qu'ils semaient partout le chaos en s'enorgueillissant » (Glossa ordinaria, $\mathrm{f}^{\mathrm{0}} 332$, nous traduisons. Cf. Augustinus, Epistula CLXIX, I, 2, p. 612, 1. 11 ; p. 613, 1. 1).

50 "EMULAMINI SPIRITUALIA, id est laudo ut spiritualia desideretis dona, sed post caritatem MAGIS hoc desiderandum est, UT PROPHETETIS, id est predicetis quia predicatio magis uersatur circa fructum caritatis ». "RECHERCHEZ CE QUI EST SPIRITUEL, c'est-à-dire je vous exhorte à aspirer aux dons spirituels, mais après la charité c'est ceci SURTOUT qui doit être désiré, QUE VOUS PROPHÉTISIEZ, c'est-à-dire que vous prêchiez, puisque surtout la prédication repose sur le fruit de l'amour», (Paris, BnF, lat. 15601, $\mathrm{f}^{\circ} 30 \mathrm{v}^{\circ} \mathrm{a}$, nous traduisons. Cf. Glossa ordinaria, $\left.\mathrm{f}^{\mathrm{o}} 331\right)$.

51 « NAM QUI PROPHETAT HOMINIBUS LOQUITUR AD EDIFICATIONEM ut in eis pariat intelligentiam ET [AD] EXHORTATIONEM, id est ut de uirtute in uirtutem animet eos ascendere, et AD CONSOLATIONEM, id est presentis tristitie [tristtitie ms.] mitigationem pro dilata remuneratione [...]». «EN EFFET CELUI QUI 
l'exposition et dans l'interprétation des mystères relatifs au Christ et à son Église. Il établit notamment une relation entre la pratique de la prédication et le motif, également original, d'une militia Christi prenant la forme d'une expositio Scripturarum en vue d'exhorter à la lutte contre les Démons (un thème déjà présent dans la Glose). Il est intéressant de noter que, chez ce disciple d'Anselme et de Raoul, les frontières entre les deux pratiques, la pastorale et l'interprétation du texte sacré, ont donc tendance à se brouiller $^{52}$.

D'une manière générale, il est également possible de souligner que l'exégèse de Jean s'enrichit d'une composante ecclésiologique, un trait innovant par rapport à la réflexion morale des deux premiers magistri. Le thème d'une prophétie-prédication ayant pour objectif l'édification de l'Église apparaît en effet de manière récurrente, comme un leitmotiv : dans plusieurs passages du commentaire, l'auteur soutient que la sublimation de l'esprit des fidèles - obtenue par le biais de la prédication-exégèse - doit être menée afin que l'Église elle-même, dans son ensemble, reçoive sa propre édification $^{53}$.

PROPHÉTISE PARLE AUX HOMMES EN VUE DE L'ÉDIFICATION afin de produire en eux la compréhension et [DE L'] EXHORTATION, c'est-à-dire pour les exhorter à s'élever de vertu en vertu et DE LA CONSOLATION, c'est-à-dire de la mitigation de la tristesse présente pour la récompense différée » (Paris, $\mathrm{BnF}$, lat. $15601, \mathrm{f}^{\mathrm{o}} 30 \mathrm{v}^{\mathrm{o}} \mathrm{a}$, nous traduisons).

52 «QUI LOQUITUR LINGUIS SEMETIPSUM, non etiam alios sicut prophetans EDIFICAT : cum sine interpretatione faciat, non militat ad utilitatem aliorum, non militat loquela sua, SED ille QUI PROPHETAT, id est qui alijs quod loquitur de Scripturis exponit ECCLESIAM EDIFICAT [...]». "CELUI QUI PARLE EN PLUSIEURS LANGUES comme s'il prophétisait S'ÉDIFIE LUI-MÊME mais pas les autres : du moment qu'il agit sans interprétation il ne milite pas pour l'utilité des autres, il ne milite pas avec sa propre éloquence. MAIS celui QUI PROPHÉTISE, c'est-à-dire celui qui expose aux autres ce qu'il dit sur les Écritures ÉDIFIE L'ÉGLISE [...] » (Paris, BnF, lat. 15601, $\mathrm{f}^{0} 30 \mathrm{v}^{\mathrm{o}} \mathrm{a}$, nous traduisons).

53 Cette position de l'auteur est particulièrement évidente dans le cadre de l'interprétation de I Cor. XIV, 4 : «[...] SED ille QUI PROPHETAT, id est qui alijs quod loquitur de Scripturis exponit ECCLESIAM EDIFICAT, id est mentes fidelium quantum in se sublimat ». «[...] MAIS CELUI QUI PROPHÉTISE, c'est-à-dire celui qui expose aux autres ce qu'il dit sur les Écritures, ÉDIFIE L'ÉGLISE, c'est-à-dire il sublime les esprits des fidèles autant qu'il le peut » (Paris, BnF, lat. 15601, $\mathrm{f}^{\mathrm{o}} 30 \mathrm{v}^{\mathrm{o}} \mathrm{a}$, 
En revanche, comme dans la Glose, il est possible d'identifier dans ce texte l'interdiction faite aux femmes de prêcher. Cet interdit est exprimé avec vigueur dans la deuxième partie du commentaire à partir de l'auctoritas paulinienne et de la citation de Genèse III, $16^{54}$. Il nous semble

nous traduisons); dans le cadre de l'exégèse de I Cor. XIV, 12: «Quandoquidem barbari uidemini illis quibus loquimini nisi intelligatis, ideo querite ut habundetis interpretatione ad aedificationem Ecclesiae, que erit si intelliget que loquimini ». «Puisque si vous ne comprenez pas vous semblerez des barbares à ceux auxquels vous parlez, donc autant que vous le pouvez soyez riches d'une interprétation qui se fasse en vue d'une édification de l'Église ; cela aura lieu si [l'Église] comprend ce que vous dites " (Paris, BnF, lat. 15601, $\mathrm{f}^{0} 30 \mathrm{v}^{\circ} \mathrm{b}$, nous traduisons. Cf. Glossa ordinaria, $\mathrm{f}^{\mathrm{o}} 331$ ) ; dans le développement du commentaire de I Cor. XIV, 13 : «ET IDEO. Quandoquidem interpretatio ualet ad edificationem Ecclesiae, IDEO QUI LOQUITUR LINGUA ORET DEUM UT INTERPRETETUR, scilicet ut Deus det sibi donum interpretationis ». «ET DONC. Puisque l'interprétation est utile à l'édification de l'Église, DONC QUE CELUI QUI PARLE EN D'AUTRES LANGUES PRIE DIEU AFIN QU'IL SOIT INTERPRÉTÉ afin que Dieu lui donne le don de l'interprétation » (Paris, BnF, lat. 15601, $\mathrm{f}^{\mathrm{o}} 30 \mathrm{v}^{\circ} \mathrm{b}$, nous traduisons). La deuxième partie de l'interprétation du chapitre n'est pas exempte de ce genre de réflexions. Voir, par exemple, l'exégèse de I Cor. XIV, 19: «SED TAMEN VOLO LOQUI [QUINQUE], id est pauca verba MEO SENSU, ut illa intelligam et alios intelligentia ipsorum uerborum instruam, cum sim IN ECCLESIA et ad utilitatem Ecclesiae debeam tendere. QUAM DECEM MILIA VERBORUM, id est quam infinita verba LINGUA tantum». "TOUTEFOIS JE VEUX PROPHÉRER CINQ paroles, soit peu de paroles SELON MA SIGNIFICATION afin de les comprendre et dinstruire d'autres à la compréhension de ces paroles, étant donné que je suis de l'Église et que je dois m'employer à l'utilité de l'Église AUTANT QUE DIX MILLE PAROLES, c'est-à-dire autant de paroles infinies proférées seulement EN LANGUE » (Paris, BnF, lat. 15601, $\mathrm{f}^{0} 31 \mathrm{r}^{\circ} \mathrm{a}$, nous traduisons. Cf. Glossa ordinaria, $\mathrm{f}^{\mathrm{o}}$ 332). Ou encore l'exégèse de I Cor. XIV, 22 : "Linguas non debetis appetere, sed sensum appetite, quia PROPHETIAE, id est sensus prophetandi NON INFIDELIBUS sunt commisse, quia nisi aliquid credant non debent eis aliquid exponi, SED FIDELIBUS, ut per eas edificentur». "Vous ne devez pas désirer les langues, désirez plutôt le sens, puisque LES PROPHÉTIES, c'est-à-dire les sens du fait de prophétiser, ont été confiées NON PAS AUX NON CROYANTS, qui, ne croyant pas, ne doivent rien exposer aux autres, MAIS AUX FIDĖLES, afin qu'ils soient édifiés grâce à elles ") (Paris, $\mathrm{BnF}$, lat. 15601, $\mathrm{f}^{0} 31 \mathrm{r}^{\circ} \mathrm{b}$, nous traduisons. Cf. Glossa ordinaria, $\mathrm{f}^{\mathrm{o}} 332$ ). Le commentaire de $I$ Cor. 14, 28 constitue le dernier exemple relatif au chap. XIV : "SI AUTEM NON FUERIT INTERPRES qui possit exponere, TACEAT ille loquens linguis IN ECCLESIA, ubi nichil est loquendum nisi ad edificationem ». "MAIS S'IL N'Y A PAS EU UN INTERPRÈTE en mesure de savoir exposer, QUE SE TAISE celui qui parle en différentes langues À L'ÉGLISE, où rien ne doit être proféré si ce n'est en vue de l'édification» (Paris, BnF, lat. $15601, \mathrm{f}^{\mathrm{o}} 31 \mathrm{r}^{\circ} \mathrm{b}$, nous traduisons. Cf. Glossa ordinaria, $\left.\mathrm{f}^{\mathrm{o}} 332\right)$.

54 «MULIERES. (in mg. : quia dixerat : OMNES DISCANT, OMNES EXHORTENTUR) Ne mulieres uellent loqui et interpretari, subdit quasi : "Licet dixerim: "Omnes 
que ces avertissements récurrents de la part de Gratiadei sont importants et qu'ils ne doivent pas être interprétés uniquement dans un sens historicolittéral, mais bien le cadre d'une exégèse tournée vers l'actualité de l'époque : de même et encore plus que le texte d'Anselme et Raoul, le texte de Jean se situe en effet dans le contexte de la vive polémique antihérétique qui caractérise la France durant ces années et qui se montre défavorable à une prédication féminine. Dans ce contexte, le commentaire de I. Cor. XIV, 15 nous semble particulièrement intéressant, dans la mesure où il condamne, à travers la figure de l'Apôtre, une prédication populaire et improvisée : «[...] il montre que le peuple ne doit pas prononcer un

potestis prophetare, tamen MULIERES TACEANT IN ECCLESIA". Vel ita: MULIERES TACEANT IN ECCLESIA apud uos SICUT DOCEO IN OMNIBUS ECCLESIJS SANCTORUM tacere eas. NON ENIM PERMITTITUR a me, uel ab aliquo predicatore ILLIS mulieribus LOQUI in aecclesia, SED precipitur SUBDITAS ESSE, SICUT ETIAM LEX DICIT. In Genesi dicit Dominus : SUB VIRI POTESTATE ERIS ET IPSE DOMINABITUR TIBI. SI QUID AUTEM VOLUNT ipse mulieres DISCERE quod non bene intellexerint in ecclesia, privatim VIROS SUOS INTERROGENT DOMI. TURPE ENIM EST MULIERI LOQUI IN ECCLESIA, que semper debet esse verecunda eo, quod primum peccatum intravit in mundum per mulierem. In signum cuius uerecundiae semper eas tacere oportet saltim in aecclesia dum illi in quo peccaverunt se representant ». "LES FEMMES. (puisqu'il avait dit : QUE TOUS ENSEIGNENT, QUE TOUS EXHORTENT). Afin que les femmes ne veuillent pas parler et interpréter, il ajoute pour ainsi dire : "Bien que j'aie dit "Vous pouvez tous prophétiser, toutefois, QUE LES FEMMES GARDENT LE SILENCE DANS LES ÉGLISES". Ou bien ainsi : QUE LES FEMMES GARDENT LE SILENCE DANS LES ÉGLISES auprès de vous TOUT COMME JE VOUS ENSEIGNE qu'elles gardent le silence DANS TOUTES LES ÉGLISES DES SAINTS. IL N'EST EN EFFET PAS CONSENTI par moi ou par tout prédicateur À CES femmes DE PARLER dans les églises, MAIS il est ordonné QU'ELLES SOIENT SOUMISES, COMME LE PRESCRIT AUSSI LA LOI. Dans le livre de la Genèse, le Seigneur dit: TU SERAS SOUS L'AUTORITÉ DE L'HOMME ET IL TE DOMINERA. MAIS SI les femmes VEULENT ENSEIGNER QUELQUE CHOSE qu'elles n'ont pas bien compris à l'église, QU'ELLES INTERROGENT EN PRIVÉ LEUR ÉPOUX. IL EST EN EFFET INFÂME POUR UNE FEMME DE PARLER À L'ÉGLISE, elle qui doit toujours être réservée à cause du fait que le premier péché est apparu dans le monde à cause de la femme. En signe de cette discrétion, il est opportun qu'elles gardent toujours le silence à l'église tant qu'elles se présentent devant Celui envers lequel elles ont péché » (Paris, BnF, lat. 15601, $\mathrm{f}^{\mathrm{o}} 31 \mathrm{v}^{\mathrm{o}} \mathrm{a}$, nous traduisons). 
discours sans le comprendre, la compréhension devant plutôt être dans le chant des psaumes; et cela seulement est pertinent pour son édification $»^{55}$.

\section{L'Anonyme des Glosulae glosularum}

Le dernier texte que nous souhaitons aborder est le commentaire du chap. XIV de la I ìre Épître aux Corinthiens des Glosulae glosularum, une œuvre plutôt importante dont nous préparons l'édition critique ${ }^{56}$. D'une manière plus générale, le texte se situe dans le cadre d'un commentaire des Épîtres de Paul dû à la contribution exégétique d'un illustre anonyme, qui réutilise la Glose ordinaire sous une forme principalement indirecte, en présentant les Glosulae epistolarum Pauli de notre Jean de Poitiers comme sa source principale. C'est justement ce fait, ainsi que l'emploi constant du commentaire des Épîtres pauliniennes d'Hervé de Bourg-Dieu ${ }^{57}(\dagger 1150$ env.) et une référence à la Vita sancti Caesarii Episcopi Arelatensis ${ }^{58}$ via Aimone d'Auxerre ${ }^{59}$ qui nous ont permis de dater notre texte de la deuxième moitié $\mathrm{du} \mathrm{XII}^{\mathrm{e}}$ siècle et de le localiser sur le territoire poitevin. Nous pouvons donc parler, d'une manière plus générale, d'une école théologique de Poitiers qui fait référénce, au cours du XII siècle, à la contribution récente des frères de Laon.

D'une manière générale, le commentaire du chap. XIV de l'Anonyme n'a pas pour ambition d'innover par rapport à sa source principale, Jean.

55 «[...] montre que le peuple ne doit pas lever sa propre prière sans discernement tandis qu'une compréhension doit être dans le chant des psaumes; cela seulement est important pour son édification » (nous traduisons).

${ }^{56}$ Nous nous appuyons ici sur notre transcription du manuscrit Florence, Laurenziana, plut. 20.23, $\mathrm{f}^{\mathrm{o}} 242 \mathrm{r}^{\mathrm{o}} \mathrm{a}-\mathrm{f}^{\mathrm{o}} 316 \mathrm{r}^{\mathrm{o}} \mathrm{b}$.

${ }^{57}$ Hervei Burgidolensis monachi In epistolam I ad Corinthios, Patrologie latine, t. 181, Lutetiae, 1854, col. 813-1002.

58 Sancti Caesarii Vita ab eius familiaribus scripta, in Sancti Caesarii episcopi Arelatensis opera omnia nunc primum collecta, t. II : Opera varia, éd. Germain Morin, Maretioli, 1942, p. 296-345.

${ }^{59}$ Haymonis Halberstadensis Episcopi, In epistolam I ad Corinthios, Patrologie latine, t. 117, Lutetiae, 1852, col. 507-606. 
Toutefois, de par son réemploi de l'Ambrosiaster, le texte ne met pas l'accent uniquement sur la prédication, mais également sur la prophétie en tant qu'exégèse et sur les prophètes en tant qu'interprètes des Écritures ${ }^{60}$. L'auteur reconnaît les contenus de la prophétie dans les mystères du Christ et de l'Église, mais surtout dans l'exposition des Écritures qui forment les coutumes $^{61}$. À travers le commentaire de Jean, c'est cet aspect moral qui apparaît finalement dominant dans les textes de l'Anonyme : la prédication est supérieure au don des langues dans la mesure où elle s'adresse à l'intelligence des hommes afin d'en régler la conduite ${ }^{62}$. L'activité du

${ }^{60}$ " "Prophetas" interpretes dicit Scripturarum : sicut enim propheta futura dicit quae nesciuit, ita et hic - dum Scripturarum sensu qui multis occultus est manifestat dicitur "prophetare" ". " Il définit comme "prophètes" les interprètes des Écritures : tout comme en effet le prophète fait référence à des évènements futurs qu'il n'a pas connus, ainsi en est-il ici de même - pendant qu'il émet des révélations par le biais du sens des Écritures qui est caché à beaucoup - cela est dit "prophétiser" " (Florence, Laurenziana, plut. 20.23, $\mathrm{f}^{\mathrm{0}} 302 \mathrm{r}^{\circ} \mathrm{b}$, nous traduisons. Cf. Ambrosiaster, Ad Corinthios prima XIV, 4, p. 150, 1. 22-25).

${ }^{61}$ "NUNC, id est cum sitis in fide VENERO AD VOS IN REVELATIONE, id est potius in expositione alicuius reuelationis. "Revelationem" uocat ubi per figuras et quasdam imagines revelata sunt quaedam mysteria Christi et Ecclesiae, ut Apocalipsin Iohannis. AUT IN SCIENTIA, id est in expositione eorum quae ad instructionem fidei Christi pertinent, scilicet ut instruam vos de Trinitate et unitate divinitatis [...] AUT IN PROPHETIA, ut exponam vobis [per] Scripturas que de futuris agit, ut haec: DEUS AB AUSTRO VENIET [Hab. III, 3] et de adventu Antichristi. Aut, nisi loquar IN DOCTRINA, id est in expositione Scripturarum quae mores informant, nullam utilitatem habebitis ex mea locutione linguarum nisi aliquid horum vos docuerim ». "MAINTENANT, c'est-à-dire tant que vous êtes dans la foi, JE VIENDRAI À VOUS EN RÉVÉLATION, c'est-à-dire plutôt dans l'exposition d'une certaine révélation. Il utilise le terme "révélation" au moment où, à travers les figures et certaines images, sont révélés des mystères donnés relatifs au Christ et à l'Église, comme l'Apocalypse de Jean. OU EN SCIENCE, soit dans l'exposition de ce qui est pertinent à la doctrine de la foi de Christ, clairement pour vous instruire sur la Trinité et l'unité de la divinité [...] OU DANS LA PROPHÉTIE, pour vous exposer [à travers] les Écritures ce qui traite du futur, comme ceci: DIEU VIENDRA DU VENT et sur l'avènement de l'Antéchrist. Ou bien, si je ne parlerai pas EN DOCTRINE, c'est-à-dire dans l'exposition des Écritures qui forment les coutumes, vous n'aurez aucune utilité du fait que je parle en d'autres langues si je ne vous aurai pas enseigné quelque chose de tout cela» (Florence, Laurenziana, plut. 20.23, $\mathrm{f}^{\mathrm{o}} 302 \mathrm{v}^{\mathrm{o}} \mathrm{a}$, nous traduisons. $C f$. Paris, $\mathrm{BnF}$, lat. $15601, \mathrm{f}^{0} 30 \mathrm{v}^{\mathrm{o}} \mathrm{a}-\mathrm{f}^{\mathrm{0}} 30 \mathrm{v}^{\mathrm{o}} \mathrm{b}$ ).

62 " [...] QUIA QUI LOQUITUR LINGUA, subaudi ignota, NON HOMINIBUS, id est hominum intelligentie LOQUITUR, SED DEO, id est ad honorem Dei qui dedit ipsam linguam per quam loqueretur magnalia Eius». «[...] CAR CELUI QUI PARLE EN UNE LANGUE, sous-entendu inconnue, NE PARLE PAS AUX 
prédicateur vise en effet à favoriser chez les autres la compréhension, une sorte d'ascèse morale, la consolation pour la récompense tardive ${ }^{63}$, ou bien - notre source est ici Hervé de Bourg-Dieu - la consolation des péchés commis : «Vel CONSOLATIONEM, ne desperent propter suam iniquitatem preteritam, quia veniam consequentur $»^{64}$.

«Celui qui prophétise édifie ceux qui l'écoutent en vue des bonnes œuvres ${ }^{65}$, précise l'Anonyme. Cela doit se réaliser également dans la perspective d'une militia Christi, d'une lutte engagée au nom du Christ. Il s'agit d'une expositio Scripturarum qui se révèle conçue au sein de la communauté des fidèles et en vue de leur édification ${ }^{66}$. L'ultime horizon

HOMMES, c'est-à-dire à la compréhension des hommes, MAIS À DIEU, c'est-àdire en hommage à Dieu qui a concédé jusqu'à la langue dans laquelle prononcer ses louanges » (Florence, Laurenziana, plut. 20.23, $\mathrm{f}^{\circ} 301 \mathrm{v}^{\circ} \mathrm{b}-\mathrm{f}^{\mathrm{o}} 302 \mathrm{r}^{\circ} \mathrm{a}$, nous traduisons. $C f$. Paris, BnF, lat. 15601, $\left.\mathrm{f}^{\mathrm{o}} 30 \mathrm{v}^{\mathrm{o}} \mathrm{a}\right)$.

${ }^{63}$ «Ideo iterum magis aemulamini prophetare quam loqui linguis. Nam qui prophetat hominibus loquitur ad edificationem, ut in eis pariat intellectum et ad exhortationem, id est de uirtute in uirtutem eos animat [animatur ms] ascendere et ad consolationem, id est presentis tristitiae mitigationem pro dilata remuneratione $»$. «Donc de nouveau aspirez à prophétiser plutôt qu'à parler des langues. En effet, celui qui prophétise pour les hommes parle pour l'édification, pour produire en ceuxlà la compréhension, et pour l'exhortation, c'est-à-dire il les incite à passer de vertu en vertu, et pour la consolation, c'est-à-dire l'adoucissement de la tristesse présente pour la récompense différée » (Florence, Laurenziana, plut. 20.23, f $\mathrm{f}^{\mathrm{O}} 302 \mathrm{r}^{\circ} \mathrm{a}$, nous traduisons. $C f$. Paris, $\mathrm{BnF}$, lat. $\left.15601, \mathrm{f}^{\mathrm{o}} 30 \mathrm{v}^{\mathrm{o}} \mathrm{a}\right)$.

${ }^{64}$ Hervei Burgidolensis monachi In Epistola I ad Corinthios, col. 959A. « [...] ou bien LA CONSOLATION, afin qu'ils ne perdent pas espoir à cause de leur iniquité passée, car ils obtiendront le pardon » (Florence, Laurenziana, plut. 20.23, f $\mathrm{f}^{\mathrm{o}} 302 \mathrm{r}^{\circ} \mathrm{a}$, nous traduisons. $C f$. Paris, $\mathrm{BnF}$, lat. $\left.15601, \mathrm{f}^{\mathrm{o}} 30 \mathrm{v}^{\mathrm{o}} \mathrm{a}\right)$.

65 «Ille qui prophetat edificat suos auditores in bona operatione». "Celui qui prophétise édifie ceux qui l'écoutent dans la bonne œuvre » (Florence, Laurenziana, plut. 20.23, $\mathrm{f}^{\mathrm{o}} 302 \mathrm{r}^{\mathrm{o}} \mathrm{a}$, nous traduisons. Cf. Ambrosiaster, Ad Corinthios prima XIV, 4 , p. $150,1.21)$.

${ }^{66}$ «[...] sed ille qui loquitur lingua misteria Dei et non exponit semet ipsum tantum et non alios edificat, id est loquela sua non militat ad utilitatem aliorum. Sed ille qui prophetat, id est qui aliis quod loquitur de Scripturis exponit Ecclesiam edificat, id est mentes fidelium quantum in se est sublimat ». " [...] mais celui qui révèle en une langue les mystères de Dieu et ne les expose pas édifie uniquement lui-même et non les autres, c'est-à-dire qu'il ne milite pas avec son éloquence pour l'utilité des autres. $\mathrm{Au}$ contraire, celui qui prophétise, c'est-à-dire celui qui expose aux autres en relation aux Écritures, édifie l'Église, c'est-à-dire il exalte les esprits des fidèles autant que ses capacités le permettent » (Florence, Laurenziana, plut. 20.23, $\mathrm{f}^{\circ} 302 \mathrm{r}^{\circ} \mathrm{a}-\mathrm{f}^{0} 302 \mathrm{r}^{\circ} \mathrm{b}$, nous traduisons. $C f$. Paris, BnF, lat. $\left.15601, \mathrm{f}^{\mathrm{o}} 30 \mathrm{v}^{\mathrm{o}} \mathrm{a}\right)$. 
moral de la prophétie-prédication-exégèse se situe ainsi dans une perspective de type ecclésiologique dans le cadre de laquelle les figures des exégètes prennent tout leur relief. L'Anonyme les définit comme «les phares » de l'Église : «Ideo dicit AUT TRES ne occuparent diem loquentes linguis et interpretes illorum et non haberent prophete tempus disserendi Scripturas, qui sint ECCLESIAE ILLUMINATORES ${ }^{67}$. Dans la conclusion de son texte, qui fait une dernière fois référence au commentaire de Jean, l'Anonyme revient lui aussi sur la prescription paulinienne relative au silence des femmes à l'église, en l'interprétant comme une forme d'interdit concernant la pratique de l'exégèse même :

Ne mulieres vellent loqui et interpretari subdit, reprimens eas in hoc quoque quasi dicat: 'Licet dixerim: OMNES POTESTIS PROPHETARE, tamen mulieres in ecclesia taceant. Vel ita : apud vos taceant mulieres in ecclesia sicut doceo in omnibus ecclesiis sanctorum eas tacere: non enim permittitur eis loqui ${ }^{68}$.

L'auteur rappelle encore une fois le rôle de soumission de la femme et les responsabilités assumées par elle lors du péché originel ${ }^{69}$.

${ }^{67}$ « Donc il dit OU BIEN TROIS également afin que les interprètes n'occupent pas la journée en parlant en langues et que les prophètes n'aient pas le temps de discuter des Écritures, en sorte d'être les phares de la communauté ecclésiale » (Florence, Laurenziana, plut. 20.23, $\mathrm{f}^{0} 305 \mathrm{v}^{\circ} \mathrm{b}$, nous traduisons. Cf. Ambrosiaster, Ad Corinthios prima, XIV, 27, p. 159, 1. 3-6).

${ }^{68}$ «Afin que les femmes ne veuillent pas parler et interpréter, il ajoute en les réprimant en cela également, pour ainsi dire: "Bien que j'aie dit VOUS POUVEZ TOUS PROPHÉTISER, toutefois que les femmes gardent le silence dans les églises". Ou ainsi : "que les femmes gardent le silence à l'église à côté de vous tout comme j'enseigne qu'elles se taisent dans toutes les églises des saints : en effet il ne leur est pas permis de parler" » (Florence, Laurenziana, plut. 20.23, $\mathrm{f}^{\mathrm{o}} 306 \mathrm{r}^{\circ} \mathrm{b}$, nous traduisons. $C f$. Paris, $\mathrm{BnF}$, lat. $\left.15601, \mathrm{f}^{\mathrm{o}} 31 \mathrm{v}^{\mathrm{o}} \mathrm{a}\right)$.

69 «Ideo precipio ut taceant quia non permittitur illis ab ullo predicatore loqui in ecclesia, sed precipitur esse subditas uiris sicut etiam Lex dicit. In Genesi enim dicit Dominus : SUB VIRI POTESTATE ERIS ET IPSE DOMINABITUR TIBI [...] turpe est mulieri loqui in ecclesia, quae semper debet esse verecunda eo, quod primum peccatum per mulierem in mundum intravit, in signum culpae cuius semper eas tacere oportet saltem in ecclesia [ecclesiam ms.], dum illis in quos peccaverunt se presentant». «Donc j'ordonne qu'elles gardent le silence puisqu'il ne leur est permis par aucun prédicateur de parler dans les églises, mais il est ordonné qu'elles 
Dans un contexte dont est de nouveau catégoriquement exclue la moindre innovation institutionnelle, l'Anonyme rappelle que ceux qui peuvent comprendre les réalités spirituelles sont ceux qui sont illuminés par le feu de l'Esprit-Saint, mais aussi les exégètes dépositaires ipso facto de la science scripturaire :

SI QUIS VIDETUR PROPHETA ESSE. Ideo iterum verbum quod predico tenere debetis, QUIA QUAE SCRIBO VOBIS MANDATA DEI SUNT et oportet ut ita cognoscat esse. SI QUIS inter vos VIDETUR ESSE PROPHETA, id est aliquis habens scientiam ut abdita Scripturarum aliis exponat. AUT si quis uidetur inter vos SPIRITUALIS esse qui habeat cor illuminatum igne (in $m g$. : sancti) Spiritus, et ideo possit spiritualia capere etiam si nesciat aliis exponere $[\ldots]^{70}$.

Comme dans la réflexion d'Anselme et de Raoul, la noncompréhension des mandata divina impliquera la non-reconnaissance du pécheur au moment du Jugement universel et son exclusion du groupe des élus : « Si quis haec mandata cognoscit esse Dei a Deo cognoscetur, sed si quis ignorat hec mandata esse Dei, id est si neglegit ea quasi non sint Dei,

soient soumises aux maris tout comme le prescrit également la Loi. Le Seigneur dit en effet dans la Genèse : TU SERAS SOUS L'AUTORITÉ DE L'HOMME ET IL TE DOMINERA [...] il est infâme pour la femme de parler dans les églises, elle qui doit toujours rester réservée à cause du fait que le premier péché s'est produit dans le monde à cause de la femme. Comme signe de cette faute il est toujours opportun qu'elles gardent le silence dans les églises tant qu'elles se présentent à ceux contre lesquels elles ont péché » (Florence, Laurenziana, plut. 20.23, $\mathrm{f}^{\mathrm{0}} 306 \mathrm{r}^{\circ} \mathrm{b}-\mathrm{f}^{\mathrm{0}} 306 \mathrm{r}^{\mathrm{o}} \mathrm{a}$, nous traduisons. $C f$. Paris, $\mathrm{BnF}$, lat. $\left.15601, \mathrm{f}^{\mathrm{o}} 31 \mathrm{v}^{\mathrm{o}} \mathrm{a}\right)$.

70 «SI QUELQU'UN SEMBLE ÊTRE PROPHÈTE. Donc de nouveau vous devez comprendre la parole que je prêche, PUISQUE CE QUE JE VOUS ÉCRIS EST ORDRE DE DIEU et il est opportun que l'on sache qu'il en est ainsi. SI QUELQU'UN entre vous SEMBLE ÊTRE PROPHÉTE, c'est-à-dire quelqu'un qui possède la science pour exposer à d'autres les secrets des Écritures, OU BIEN si quelqu'un entre vous semble être SPIRITUEL en ce qu'il a le cœur illuminé par le feu de l'Esprit (saint) et peut donc saisir les significations spirituelles, même s'il ne sait pas les exposer à d'autres [...] » (Florence, Laurenziana, plut. 20.23, $\mathrm{f}^{0} 306 \mathrm{v}^{\mathrm{o}} \mathrm{a}$, nous traduisons. $C f$. Paris, $\mathrm{BnF}$, lat. 15601, $\mathrm{f}^{\mathrm{o}} 31 \mathrm{v}^{\mathrm{o}} \mathrm{a} ;$ Ambrosiaster, Ad Corinthios prima XII, 8, p. 134, 1. 10). 
quamuis uideatur spiritualis esse, a Deo tamen ignorabitur $[\ldots] \gg{ }^{71}$. La citation de Mathieu conclut le commentaire de ce chapitre par une claire référence à la prédestination : " [...] unde in Euangelio: "AMEN AMEN dico uobis [...] nescio uos", id est non noui uos esse inter predestinatos $»^{72}$.

L'intérêt suscité par une série d'auteurs du XIII siècle - Thomas d'Aquin et Guillaume d'Auxerre, Hugues de Saint-Cher et Philippe le Chancelier, Albert le Grand et Bonaventure - et par leur réflexion systématique, explique que peu d'attention a été accordée, sur le plan historico-critique, à de nombreux auteurs plus anciens, qui sont souvent restés inédits. Leur exégèse porte entre autres sur le commentaire du

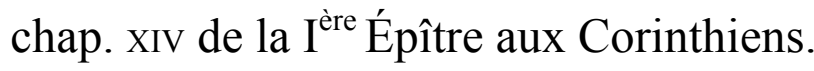

Le commentaire de la Glose ordinaire, par ailleurs diffusée sous forme anastatique à partir de l'édition de 1492 d'Alfred Rusch, tire parti de la réflexion sur la prophétie déjà proposée par Augustin afin de proposer un schéma anthropologique et cognitif bipartite. Le spiritus et un type de connaissance portant sur les images se voient opposés à la mens et au niveau de connaissance supérieure, caractéristique de la prophétie, qu'elle implique. Ce même schéma, enrichi par une lecture parfois directe de l'évêque d'Hippone et par une importante composante de réflexion à caractère ecclésial, sera également proposé par Gilbert de la Porrée dans le cadre de sa Media Glossatura.

${ }^{71}$ Si quelqu'un reconnaît que ces ordres sont de Dieu il sera reconnu par Dieu, mais si quelqu'un ignore que ces ordres sont de Dieu, c'est-à-dire s'il les néglige comme s'ils n'étaient pas de Dieu, il pourra sembler spirituel mais il sera toutefois ignoré par Dieu » (Florence, Laurenziana, plut. 20.23, $\mathrm{f}^{0} 306 \mathrm{v}^{\circ} \mathrm{b}$, nous traduisons. Cf. Paris, $\mathrm{BnF}$, lat. 15601, $\left.\mathrm{f}^{\mathrm{o}} 31 \mathrm{v}^{\mathrm{o}} \mathrm{a}\right)$.

72 «[...] d'où, dans l'Évangile : Je vous dis "AMEN AMEN [...] je ne vous connais pas", c'est-à-dire que je ne vous ai pas reconnus comme étant dans les prédestinés » (Florence, Laurenziana, plut. 20.23, $\mathrm{f}^{\mathrm{o}} 306 \mathrm{v}^{\circ} \mathrm{b}$, nous traduisons. Cf. Paris, BnF, lat. $\left.15601, \mathrm{f}^{\mathrm{o}} 31 \mathrm{v}^{\mathrm{o}} \mathrm{a}\right)$. 
Les Glosulae epistolarum Pauli de Jean, en revanche, présentent une exégèse dénuée de toute réflexion spéculative. Elles reprennent le matériel proposé par la glose interlinéaire et marginale dans un commentaire continu qui se veut avant tout une réflexion sur une pastorale - et une exégèse - délimitées par un cadre d'ordre ecclésiologique et moral. Jean identifie en effet la prophétie avec la pratique de la prédication relative au Christ et à son Église ainsi qu'avec l'interprétation des Écritures saintes, le tout dans une conception d'une Église institutionnelle et en vue de son édification.

L'Anonyme poitevin, en s'appuyant cette fois-ci sur l'exégèse de Jean de Poitiers même et sur celle de l'Ambrosiaster, met avant tout l'accent sur une prophétie-prédication qu'il considère comme relative au Christ et à son Église, mais aussi à une expositio Scripturarum qu'il conçoit dans le cadre de la communauté des fidèles et en vue de leur édification morale. L'attention de l'auteur finit d'ailleurs par se focaliser sur la prophétie en tant que forme d'exégèse et sur les prophètes en tant qu'interprètes des Écritures au sein de la communauté ecclésiale. Ces derniers, que l'Anonyme ne définit pas par hasard comme les illuminatores Ecclesiae, sont considérés comme les véritables dépositaires de la science scripturaire.

Une réflexion d'ordre plus général est enfin nécessaire en ce qui concerne la morale féminine de nos auteurs, tous influencés par le texte de la Glose. Dans l'ensemble de ces textes, le phénomène prophétique (c'està-dire, pour nos exégètes, la prédication) est en effet limité aux hommes. Aucune intervention active n'est autorisée aux femmes: l'interdiction absolue est légitimée dans chaque cas par la responsabilité assumée par la femme au moment du péché originel, en vertu de la citation de Genèse III, 16 et au nom de la succession apostolique. Elle s'explique probablement par le contexte de la vive polémique antihérétique qui occupe la France 
entre le $\mathrm{XI}^{\mathrm{e}}$ et le $\mathrm{XII}^{\mathrm{e}}$ siècles et par les nouvelles attitudes sociales des masses.

Présentée à partir de la réflexion d'Augustin comme un phénomène épistémologique, la prophétie devient donc progressivement pour nos auteurs une prédication (édifiante) au sein de l'Église, en n'impliquant cependant aucune forme de participation féminine. Pourtant la dernière interprétation proposée par l'Anonyme à l'égard de la prophétie, celle d'une expositio Scripturarum qui serait propre aux exégètes illuminatores Ecclesiae, nous semble peut-être la plus intéressante dans le cadre de ces textes, dans la mesure où elle est clairement autoréférentielle. 